\title{
SUMOylation of RIG-I positively regulates the type I interferon signaling
}

\author{
Zhiqiang $\mathrm{Mi}^{1,2}$, Jihuan $\mathrm{Fu}^{2}$, Yanbao Xiong ${ }^{2}$, Hong Tang ${ }^{2}$ \\ ${ }^{1}$ Center for Molecular Immunology, Institute of Microbiology, Chinese Academy of Sciences, Beijing 100101, China \\ 2 Key Laboratory of Infection and Immunity of Chinese Academy of Sciences, Institute of Biophysics, Beijing 100101, China \\ $\triangle$ Correspondence: tanghong@moon.ibp.ac.cn \\ Received December 29, 2009 Accepted January 21, 2010
}

\begin{abstract}
Retinoic acid-inducible gene-I (RIG-I) functions as an intracellular pattern recognition receptor (PRR) that recognizes the 5'-triphosphate moiety of single-stranded RNA viruses to initiate the innate immune response. Previous studies have shown that Lys63-linked ubiquitylation is required for RIG-I activation and the downstream anti-viral type I interferon (IFN-I) induction. Herein we reported that, RIG-I was also modified by small ubiquitin-like modifier-1 (SUMO-1). Functional analysis showed that RIG-I SUMOylation enhanced IFN-I production through increased ubiquitylation and the interaction with its downstream adaptor molecule Cardif. Our results therefore suggested that SUMOylation might serve as an additional regulatory tier for RIG-I activation and IFN-I signaling.
\end{abstract}

KEYWORDS RIG-I, SUMOylation, type I interferon, innate immunity

\section{INTRODUCTION}

Type I interferons (IFN-I) play a key role in mediating antiviral innate immune. Mammalian cells have developed two distinct pathways to recognize the viral nucleic acids and trigger the production of IFNs. One is mediated by Toll-like receptors (TLRs) and mainly recognizes extracellular viral RNA. The other utilizes the retinoic acid-inducible gene I (RIG-I)like helicases (RLHs), including RIG-I and melanoma differentiation-associated gene 5 (MDA5, also referred as helicard or IFNH1), to recognize the intracellular viral RNA (Yoneyama et al., 2005; Kato et al., 2006; Meylan et al., 2006). Both RIG-I and MDA5 consist of two N-terminal caspase-recruiting domains (2CARD), a central DExD/H box RNA helicase domain, and a C-terminal regulatory domain
(Kang 2002, 2004; Kato et al., 2005) with distinct substrate preference to different viruses (Kato et al., 2006). It seems that RIG-I is a sensor of short dsRNA as well as 5'ppp ssRNA, while MDA5 is activated by long dsRNA (Gitlin et al., 2006; Hornung et al., 2006; Yoneyama and Fujita, 2008). The 2CARD domain interacts with Cardif/IPS-1/MAVS/VISA to initiate the IFN-I signaling cascade (Kawai et al., 2005; Meylan et al., 2005; Seth et al., 2005; Xu et al., 2005). In this process, ubiquitylation at Lys172 of RIG-I 2CARD by an E3 ligase Trim25 is required for RIG-I activation, because targeting Trim25 inactivates RIG-I and increases replication of Sendai virus (SeV) and Vesicular Stomatitis virus (VSV) in fibroblast cells (Gack et al., 2007). Due to the essential roles in anti-viral innate immune response of the host cells, ubiquitylation of RIG-I is finely regulated by cellular factors to safeguard an appropriate activation. For example, both A20 (Lin et al., 2006) and RNF125 (Arimoto et al., 2007) negatively regulate RIG-I ubiquitylation. Atg5 or Atg12 conjugation also downregulates IFN-I production by direct association with RIG-I and Cardif (Jounai et al., 2007).

Similar to ubiquitylation, SUMOylation is a multi-step reaction that covalently conjugates a $12-\mathrm{kDa}$ small ubiquitinlike modifier (SUMO) to target proteins by a single E1activating enzyme (Aos1/Uba2, also called SAE1/2), a unique E2 conjugating enzyme (Ubc9) and an array of different E3 ligases (e.g., PIAS family and RanBP2), so as to regulate their activity, stability and subcellular localization (Hershko and Ciechanover, 1998; Hay, 2005). In contrast to ubiquitylation, Ubc9 can directly attach SUMO to its substrate in the absence of E3 ligase (Desterro et al., 1997). SUMOylation can also antagonize other post-translational modification, such that SUMOylation stabilizes IKBa through blocking its ubiquitylation at the same ubiquitin acceptor site (Desterro et al., 1998). SUMOylation is believed to regulate IFNs signaling pathway, in that virus-mediated SUMOylation of IRF3 and IRF7, which are two transcription factors for RIG-I- 
regulated IFN-I production, attenuates the activation of IFNs (Kubota et al., 2008).

Bioinformatic analysis suggested that RIG-I is abundant in lysine residues and some of them match the consensus SUMO acceptor sites. However, whether RIG-I can be SUMOylated and which role(s) of this modification might play in IFN-I signaling is still unclear. In the present work, we demonstrated that both exogenously expressed and endogenous RIG-I were modified by SUMO-1. RIG-I SUMOylation increased its Lys63 ubiquitin modification and the intermolecular interaction between RIG-I and Cardif. Reporter assays showed that modulation of Ubc9 levels altered the SUMO-1 modification of RIG-I, which well correlated with the RIG-I-driven IFN- $\beta$ production. These results implied that SUMOylation provided an additional regulation of RIG-I activation, which might crosstalk to ubiquitylation of RIG-I to orchestrate the cellular anti-viral response.

\section{RESULTS}

\section{RIG-I is modified by SUMO-1}

Bioinformatic analysis (SUMOplot Analysis Program, ABGENT) predicted that nine lysine residues in RIG-I could be potential SUMO acceptors (supplemental Fig. 1A), bearing the consensus motif of $\psi \mathrm{KxE}$ or non-consensus motif with high modification frequency (Song et al., 2004; Schwamborn et al., 2008; Xu et al., 2008). To validate these predicted SUMOylation sites, Flag-RIG-I, HA-SUMO-1 and Myc-Ubc9 were overexpressed in HEK293T cells. The immunoblotting of the whole cell lysate with anti-Flag antibody showed several characteristic band shifts with higher molecular weights, indicating the existence of post-translational modification (Fig. 1A, left panel). When the cell lysates were subjected to immunoprecipitation with anti-Flag antibody, these characteristic bands could be probed by anti-SUMO-1 antibody (Fig. 1A, right panel). In physiological condition, the expression level of RIG-I is trivial in cells (Cui et al., 2004; Imaizumi et al., 2004), but viral infection, such as by $\mathrm{SeV}$, can cause massive production of RIG-I. To enhance the SUMOylation signal of the endogenous RIG-I, SeV was used to infect HEK293T cells that had been overexpressed with HA-SUMO1 and Myc-Ubc9. Co-immunoprecipitation with anti-SUMO-1 antibody showed that the endogenous RIG-I was present in the immunoprecipitated complex and a small fraction of RIG-I was modified by SUMO-1 (Fig. 1B). The apparently unmodified RIG-I by anti-SUMO-1 antibody was also detected in the complex, which is due to the dynamic nature of reversible
B

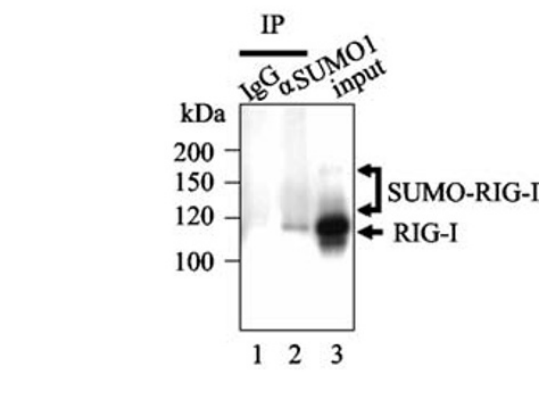

A

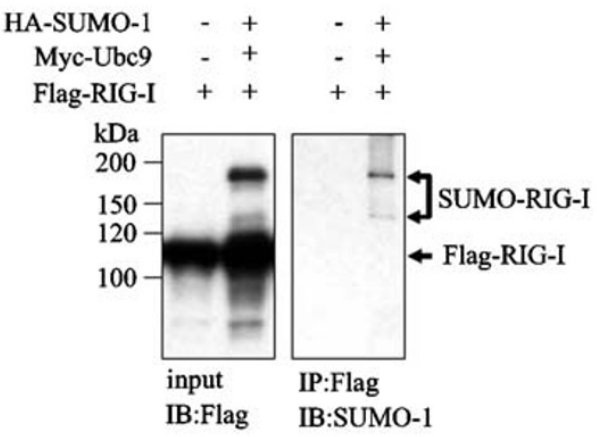

C

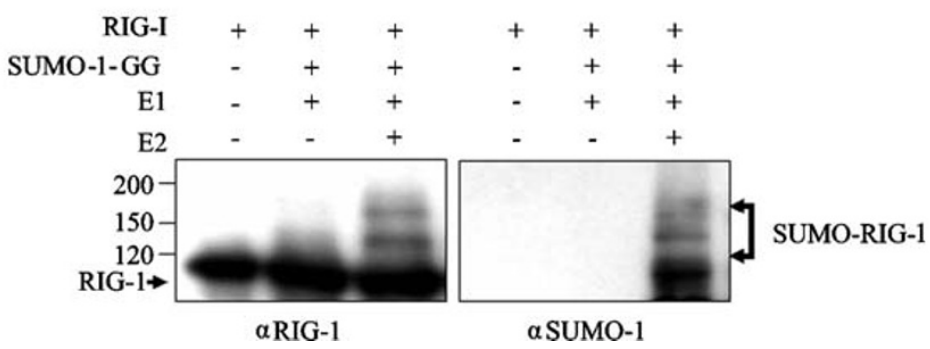

Figure 1. RIG-I was modified by SUMO-1. (A) Overexpressed RIG-I was SUMOylated. HEK293T cells were co-transfected with pCMV-HA-SUMO-1, pcDEF-Myc-Ubc9 and pEF-Flag-RIG-I, and cell lysates were immunoprecipitated with anti-Flag M2 mAb and immunoblotted with anti-Flag or anti-SUMO-1 (D-11, Santa Cruz) mAb. (B) Endogenous RIG-I was SUMOylated. HEK293T cells $\left(2 \times 10^{6}\right)$ were co-transfected with pCMV-HA-SUMO-1 and pcDEF-Myc-Ubc9. Twelve hours post-transfection, cells were infected with $20 \mathrm{HAU} \mathrm{SeV}$ for $1.5 \mathrm{~h}$, and excessive virus was washed off. Cells were then changed with fresh medium and subsequently cultured for $36 \mathrm{~h}$. Cell lysates were immunoprecipitated with anti-SUMO-1 antibody or mouse IgG1 as a negative control, and proteins were resolved in SDS-PAGE for immunoblotting with mouse anti-RIG-I antibody (Alme-1, Alexis). (C) RIG-I was SUMOylated in in vitro enzymatic assay. Recombinant RIG-I was incubated with purified SUMO-1-GG and E1/E2 enzymes for $3 \mathrm{~h}$. Then the reaction mixtures were separated by SDS-PAGE and immunoblotted with mouse anti-RIG-I or anti-SUMO-1 antibody. 
SUMOylation of the protein. Such intracellular SUMOylation of RIG-I was further confirmed by in vitro enzymatic assays, where we used purified recombinant enzymes (SAE1/SAE2 and Ubc9) and substrates (SUMO-1-GG and RIG-I) from bacteria. As indicated in Fig. 1C, in the presence of E1 and E2 enzymes, purified RIG-I was readily conjugated with SUMO-1 with the typical band shifts (Fig. 1C, left panel) that corresponded to oligomeric or polymeric SUMO-1 attachment (Fig. 1C, right panel).

\section{RIG-I interacts with Ubc9 and its SUMOylation is SUMO-1 specific}

SUMOylation requires direct interaction between Ubc9 and target proteins in order to transfer SUMO moiety from E1 enzyme. The inter-molecular interaction between RIG-I and Ubc9 was detected by co-immunoprecipitation in the presence of SUMO-1 (Fig. 2A). Furthermore, there are at least four SUMO genes (SUMO1-4) in human, with SUMO1-3 ubiquitously expressed and SUMO-4 mainly in kidney, lymph node and spleen (Guo et al., 2004). SUMO-2 and SUMO-3 are $97 \%$ identical, but share only $50 \%$ sequence identity with SUMO-1. SUMO-1 and SUMO-2/3 have distinct functions, as they are conjugated to different target proteins in vivo, while the role of SUMO-4 remains enigmatic (Geiss-Friedlander and Melchior, 2007). Up to date, a large number of target proteins were found to be modified by SUMO, but most of them were substrates of SUMO-1. SUMOplot Analysis Program predicted the potential SUMO-1 acceptation site in RIG-I. Therefore, we first investigated whether RIG-I could be modified by SUMO-1, and we also determined whether SUMO-2 and SUMO-3 are involved in RIG-I modification. In our assays, only SUMO-1 overexpression gave rise to the characteristic higher molecular weight bands, suggesting that RIG-I was a SUMO-1 acceptor (Fig. 2B).

\section{SUMOylation enhances the interaction between RIG-I and Cardif}

Although highly reversible and dynamic, a small proportion of SUMO conjugation results in significant function alteration of substrate protein through inter- or intra-molecular interaction (Geiss-Friedlander and Melchior, 2007). It has been previously shown that Lys63-linked ubiquitylation disrupts the auto-inhibitory conformation of RIG-I, which is essential for IFN-I signaling (Saito et al., 2007). In the situation of overexpression, RIG-I can be ubiquitylated by Lys63-linked ubiquitin in the absence of viral infection (Gack et al., 2007)

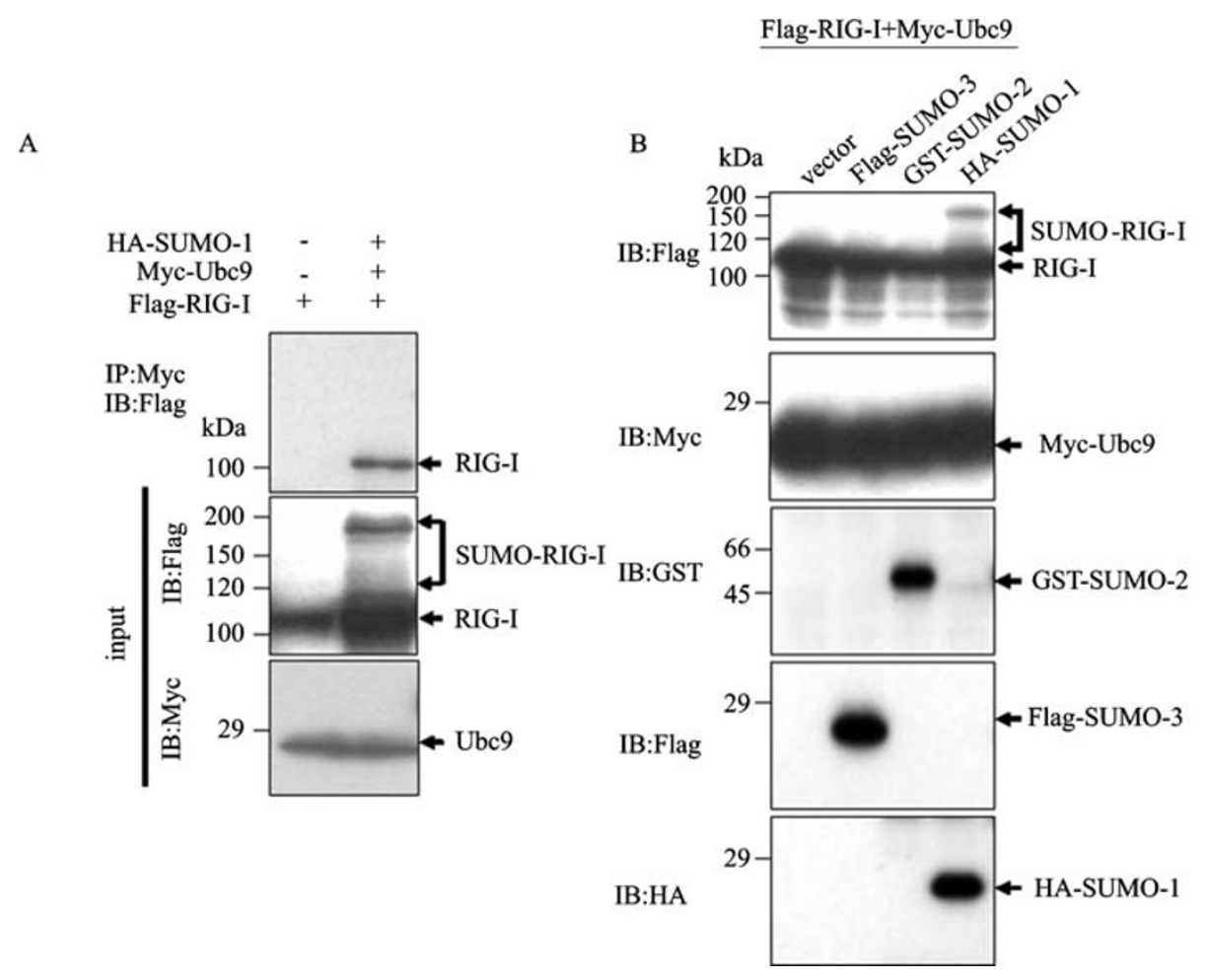

Figure 2. RIG-I interacted with Ubc9 for SUMO-1 conjugation. (A) RIG-I and Ubc9 co-immunoprecipitated. Cell lysates from Fig. 1A were immunoprecipitated with anti-c-Myc antibody (9E10, Zymed) and immunoblotted with anti-Flag or c-Myc antibody. (B) pcDEF-Myc-Ubc9 and pEF-Flag-RIG-I were co-transfected with pCMV-HA-SUMO-1, or pcDEF-GST-SUMO-2, pcDEF-FlagSUMO-3, respectively, for $48 \mathrm{~h} .2 \times 10^{5}$ cells were boiled in SDS-PAGE loading dye for 5 min before loaded into $10 \%$ SDS-PAGE. Mobility shifts of RIG-I was detected by immunoblotting with anti-Flag antibody. Expression levels of Ubc9, SUMO-1, SUMO-2 and SUMO-3 were immunoblotted with anti- c-Myc, -HA, -GST or-Flag antibody. 
and drive the production of IFN- $\beta$. A small fraction of RIG-I pool shows activation, and even in the absence of viral infection, the signaling might facilitate the maintenance of the basal level of interferon signal transduction that contributes to the rapid and massive interferon production when pathogen invades (Taniguchi and Takaoka, 2001, 2002). Change of RIG-I SUMOylation by overexpression or siRNA knockdown of Ubc9 increased or decreased RIG-I ubiquitylation, respectively (Fig. 3A and 3B). This result suggested that SUMOylation likely alters the protein folding to assist RIG-I ubiquitylation. We then determined whether RIG-I SUMOylation might affect its interaction with the adaptor Cardif. Coimmunoprecipitation assays showed that overexpression of Ubc9 significantly enhanced the interaction between RIG-I and Cardif (Fig. 3C), whereas RNAi knockdown of Ubc9 effectively decreased this inter-molecular interaction (Fig. 3D). The effect of Ubc9 knockdown was verified by RT-PCR (supplemental Fig. 1B) and this also resulted in overall reduction of SUMOylation of cellular proteins (supplemental Fig. 1C).

\section{RIG-I SUMOylation increases IFN- $\beta$ production}

Although RIG-I exists as a monomer in resting cells due to an auto-inhibitory domain (Meylan et al., 2005), the viral infection or overexpression promotes its self-association. Thus, overexpression of RIG-I can potentially initiate certain
A

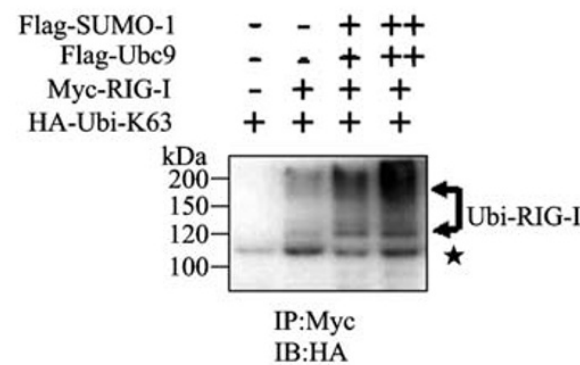

C

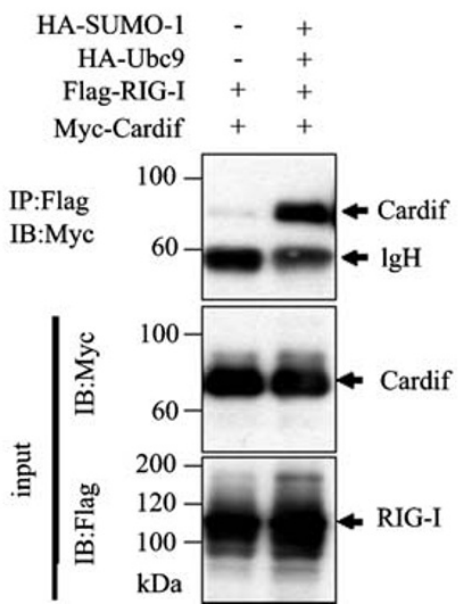

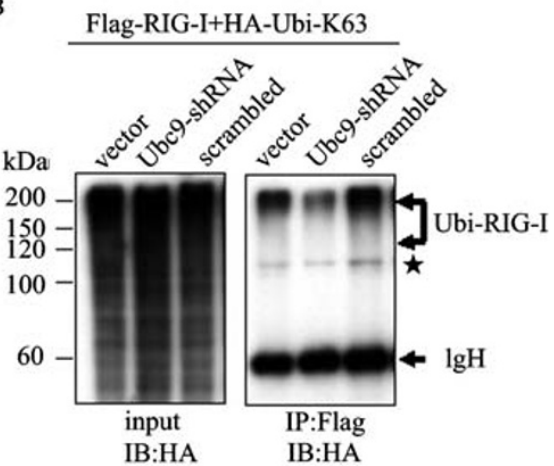

$\mathrm{D}$

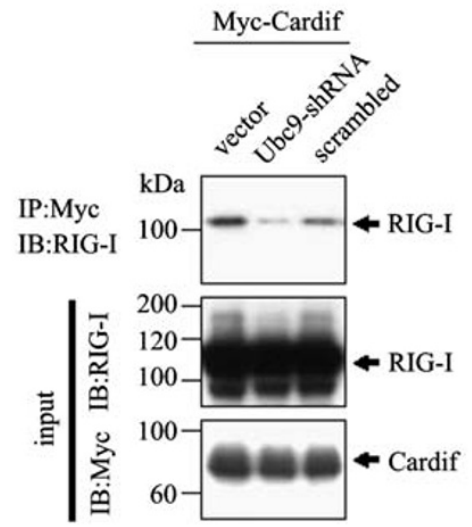

Figure 3. SUMOylation increased interaction between RIG-I and Cardif. (A) SUMOylation increased Lys63-linked ubiquitylation of RIG-I. HEK293T cells were transfected as described in Fig. 1A except that HA-Ubi expression vector was included. The cell lysates were immunoprecipitated with anti-c-Myc and immunoblotted with anti-HA mAb. The star $(\star)$ indicated non-specific bands. (B) Knockdown of Ubc9 reduced ubiquitylation of RIG-I. HeLa cells $\left(2 \times 10^{6}\right)$ were pre-infected with 2 MOI lentiviral vector, lentivirus containing Ubc9-shRNA or scrambled Ubc9-shRNA for $12 \mathrm{~h}$. Cells were then co-transfected with pEFFlag-RIG-I and pRK5-HA-Ubi-K63 for $48 \mathrm{~h}$. Co-immunoprecipitation was performed as described in Fig. 1A for detection of ubiquitylation with anti-HA antibody. The star $(\star)$ indicated non-specific bands. (C) SUMOylation enhanced inter-molecular interaction of RIG-I and Cardif. HEK293T cells were co-transfected with indicated plasmids, and cell lysates were immunoprecipitated with anti-Flag mAb and immunoblotted with anti-c-Myc mAb. The input was measured with anti-Flag or antiMyc mAb. (D) Knockdown of Ubc9 disrupted RIG-I/Cardif interaction. Endogenous Ubc9 was knocked down by lentivirus-based shRNA as described in (B). HeLa cells were then co-transfected with pCMV-Myc-Cardif for $36 \mathrm{~h}$. Interaction of endogenous RIG-I with Cardif was detected by co-immunoprecipitation as described in (C). 
downstream signaling(s) in cells (Saito et al., 2007). SUMOylation of RIG-I enhanced ubiquitylation and the subsequent inter-molecular interaction with Cardif, which inevitably would result in RIG-I activation and IFN-I induction. To prove this hypothesis, the IFN- $\beta$ reporter activities were measured in the presence of overexpressed SUMO-1 and Ubc9. Intriguingly, RIG-I-, but not Cardif-driven IFN- $\beta$ reporter activity was augmented by Ubc9 (Fig. 4A). Because RIG-I functions in the upstream of Cardif in IFN- $\beta$ signaling cascade, this result suggested that Ubc9 is specifically involved in RIG-I SUMOylation. This was further confirmed by RNAi knockdown of endogenous Ubc9, where RIG-I- but not Cardif-driven IFN- $\beta$ reporter activities were reduced (Fig. 4B). Previous reports have demonstrated that SeV infection induces IFN-I production through RIG-I activation (Gack et al., 2007). Small RNA interference of Ubc9 in HeLa cells showed that downregulation of Ubc9 caused reduced IFN- $\beta$ reporter activities (Fig. $4 \mathrm{C}$ ), which led to enhanced viral replication (Fig. 4D). Therefore, our results strongly suggested that SUMOylation actively regulates RIG-I activation, which modulates IFN-I production and resistance to viral infection.
A

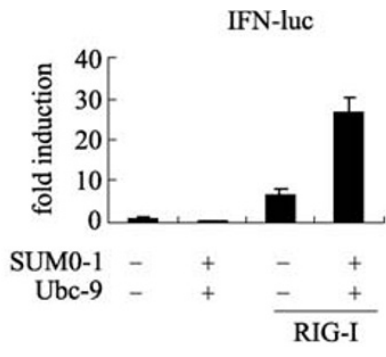

B

C
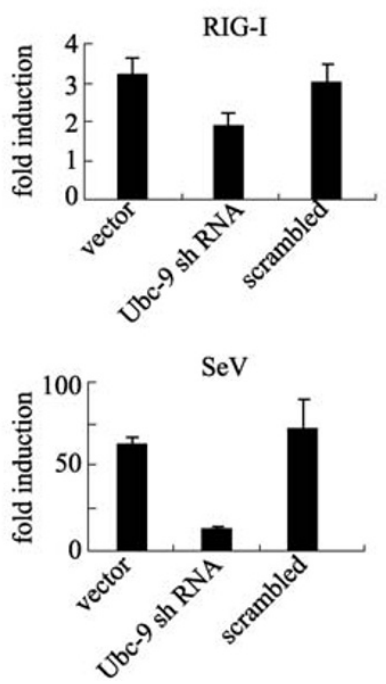

IFN-luc
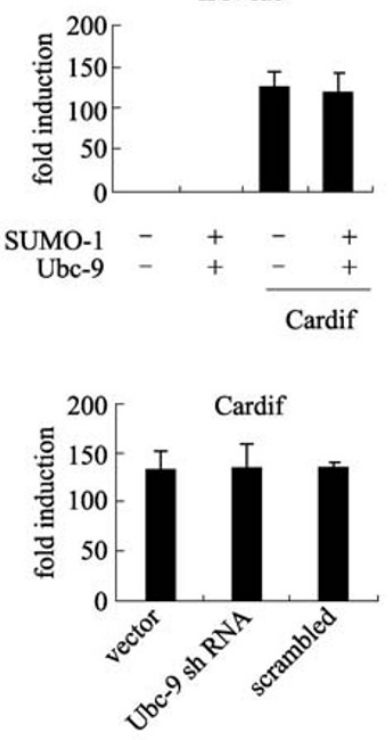

D

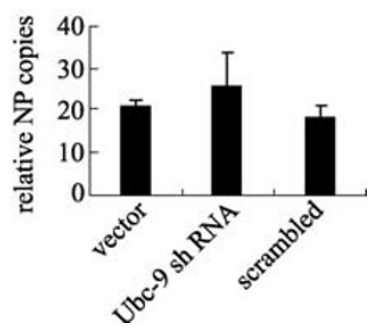

Figure 4. SUMOylation increased RIG-I-driven IFN production. (A) Ubc9 enhanced RIG-I-responsive IFN production. HEK293T cells were co-transfected with pIFN-luc, pCMV-renilla, pCMV-HA-SUMO-1, pcDEF-Myc-Ubc9, pEF-Flag-RIG-I (left panel) or pCMV-Myc-Cardif (right panel) by calcium phosphate participation. Forty-eight hours post-transfection, cells were collected and the dual-luciferase activities were measured. (B) RIG-I- but not Cardif-driven IFN signaling was affected by Ubc9 knockdown. HeLa cells were pre-infected with lentiviral vectors to knockdown endogenous Ubc9 as described in Fig. 3B. Cells were then cotransfected with pIFN-luc, pCMV-renilla, pEF-Flag-RIG-I (left panel) or pCMV-Myc-Cardif (right panel) by jetPEI reagent for $36 \mathrm{~h}$. (A) and $(B)$. The luciferase activity was determined and normalized by renilla luciferase activity. Data were presented as fold induction through dividing the luciferase activity for each sample by that for the control sample with empty vector only. Data represented the average of three independent experiments (mean $\pm \mathrm{SD}$ ). (C) Knockdown of Ubc9 inhibited SeV-responsive IFN production. Endogenous Ubc9 was knocked down by small RNA interference as described in (B), and HeLa cells were then co-transfected with pIFN-luc and internal control pCMV-renilla for $24 \mathrm{~h}$. SeV (2OHAU) was used to infect cells for $12 \mathrm{~h}$ before measuring luciferase activities. The luciferase activity was determined and normalized by renilla luciferase activity. Fold induction represents the ratio of $\mathrm{SeV}$ infection to mock infection. Data from three independent experiments were shown as mean \pm SD. (D) Knockdown of Ubc9 facilitated SeV replication. After Ubc9 knockdown as described in (C), cells were infected with SeV (20HAU) for $12 \mathrm{~h}$. Virus replication was measured by quantitative real-time RT-PCR analysis of NP gene copy numbers. Data represented the average of SeV NP gene copy number per $100 \beta$-actin copies (mean \pm SD) from three independent experiments. 


\section{DISCUSSION}

As a pivotal sensor of RNA viruses in IFN-I signaling, RIG-I activation has to be tightly regulated to ensure effective eradication of pathogens with minimal excessive inflammation. For example, to maintain the homeostasis, ubiquitylated RIG-I by Trim25 (Gack et al., 2007) needs to be downregulated by either RNF125-recruited proteasomal degradation (Arimoto et al., 2007, 2008) or ISG15 conjugation (Zhao et al., 2005; Arimoto et al., 2008; Kim et al., 2008). In the present work, we provided evidence that SUMOylation, however, might serve as an additional positive regulator in RIG-I activation, which facilitates the ubiquitylation of RIG-I and inter-molecular interaction with its mitochondrial adaptor Cardif. This positive role of RIG-I SUMOylation in IFN-I production is particular interesting, provided current evidence that SUMOylation is involved in inhibition of IFN-I signaling. For example, SUMOylation of IRF3 and IRF7 upon VSV infection inhibits IFN-I transcription activation (Kubota et al., 2008). SUMOylation of IRF2 by SUMO E3 ligase PIASy inactivates transcription of IFN-I-responsive genes (Han et al., 2008). Our finding that SUMOylation enhanced RIG-Idriven but not Cardif-driven IFN- $\beta$ reporter strongly indicated that SUMOylation in the upstream molecules can overcome its effect on the downstream IRFs, with SUMOylation of RIG-I becoming dominant in control of IFN-I production.

The existence of an auto-inhibitory conformation of RIG-I is a useful tactics for host cells since it is activated only after cells sense the invading pathogenic RNA genomes (Saito et al., 2007) and after ubiquitylation triggers the conformational unfolding (Gack et al., 2007). Interestingly, we observed that SUMOylation increased Lys63-linked ubiquitylation of RIG-I, suggesting that SUMOylation would occur upstream of ubiquitylation of RIG-I for its activation. Furthermore, RIG-I mutant (K172R) defective in ubiquitylation (Gack et al., 2007) was also SUMOylated (supplemental Fig. 2A), implying that these two types of modification did not compete for the same lysine sites. This was inconsistent previous reports that SUMOylation and ubiquitylation interfere with each other (Desterro et al., 1998; Comerford et al., 2003; Huang et al., 2003; Lin et al., 2003; Steffan et al., 2004). According to the characteristic band shift pattern, RIG-I might be SUMOylated multimerically or polymerically. After investigating the potential SUMO acceptors by mutagenesis, however, we found that these sites were not essential in mediating RIG-I modification (supplemental Fig. 2B), because SUMO modification still occurred in these RIG-I mutants. This could be explained by the variation of flanking amino acids that do not fit with the consensus motif (Hay, 2005; Anckar and Sistonen, 2007). Whereas we speculate that the SUMO acceptor sites in RIG-I might not agree with the consensus acceptor site, further mass spectrometric analysis is required to prove this hypothesis.

Post-translational and induced modifications of RIG-I by small molecule (e.g., Ubi, SUMO-1 and ISG15) present a fine regulatory network for innate cellular response to pathogens. In terms of SUMOylation, although the E2 enzyme is universal, the poll of E3 ligase is rather diverse and the modification is rather target specific (Melchior et al., 2003; Hay, 2005). It remains interesting to identify other E3 ligase that would be involved in RIG-I SUMOylation after our analysis of PIAS family E3 ligases on RIG-I modification.

\section{MATERIALS AND METHODS}

\section{Viruses, cells, plasmid constructs and transient tranfection}

Sendai virus was from Wuhan Institute of Virology, Chinese Academy of Sciences. Sendai virus was propagated in 10-day-old embryonated chicken eggs from specific-pathogen-free flocks (Beijing MERIAL Ltd.) as previously described (Mattana and Viscomi, 1998). The hemagglutination titers were measured with $1 \%$ hamster blood cell (Beijing MERIAL Ltd.).

HeLa cell and HEK293T cell were routinely maintained in minimal essential medium (MEM) supplemented with $10 \%$ fetal bovine serum (PAA), $100 \mathrm{unit} / \mathrm{mL}$ penicillin and $100 \mu \mathrm{g} / \mathrm{mL}$ streptomycin (HyClone) and cultured at $37^{\circ} \mathrm{C}$ with $5 \% \mathrm{CO}_{2}$. Cells were transiently transfected with calcium-phosphate precipitation and jetPEI (Polyplus transfection), respectively.

pEF-Flag-RIG-I (Yoneyama et al., 2004) was kindly provided by Prof. S. Akira (Osaka University, Japan), and its K/R mutant derivatives were generated by site-directed mutagenesis (Zheng et al., 2004). pCMV-Myc-RIG-I was constructed by add-on PCR to insert in between Sall and Notl sites of pCMV-Myc vector (Clontech). pcDEF-Flag-SUMO-1, pcDEF-Myc-Ubc9, pcDEF-Myc-SAE1/SAE2, pcDEF-GST-SUMO-2 and pcDEF-Flag-SUMO-3 were kind gifts from Dr. X. Peng (Chinese Academy of Medical Sciences, Beijing). pGEX6P-1-RIG-I was constructed by add-on PCR to insert RIG-I gene in between Smal and Xhol sites of pGEX-6P-1 vector (Amersham). pGEX-4T-1-SUMO-1, Ubc9, SAE1 and SAE2 were constructed by add-on PCR to insert each encoding gene in between EcoRI and Xhol sites of pGEX-4T-1 vector (Amersham). Ubiquitin expression vector pRK5-HA-Ubi-K63 containing arginine substitutions except position 63 (Lim et al., 2005) was from Dr. K. Lim (National Neuroscience Institute, Singapore). Interferon beta reporter (IFN-luc) (Guo and Cheng, 2007) were kindly provided by Prof. G. Cheng (UCLA, USA). pCMV-Flag-Ubc9 was constructed by inserting Ubc9 in between EcoRI and Xhol restriction sites of pCMV-Tag2 (Stratagene). pCMVHA-SUMO-1, pCMV-HA-Ubc9, pCMV-Myc-Cardif were constructed by cloning of each encoding gene in between EcoRI and Xhol, Xhol and Kpnl, and Sall and Notl restriction sites of $\mathrm{pCMV}$ cassette vectors (Clontech), respectively.

\section{Immunoprecipitation and immunoblotting}

Routinely, $2 \times 10^{6}$ cells were lysed in $250 \mu \mathrm{L}$ ice-cold immunoprecipitation buffer ( $50 \mathrm{mM}$ Tris-HCl, pH 7.5, $150 \mathrm{mM} \mathrm{NaCl}, 1 \%$ Nonidet P$40,0.5 \%$ sodium deoxycholate) freshly supplemented with $2 \mathrm{mM} \mathrm{N}$ ethylmaleimide (NEM) (Sigma), $1 \mathrm{mM}$ DTT (Sigma) and complete protease inhibitor cocktail (Roche). Cell lysates $(100 \mu \mathrm{g}$ proteins) were then immunoprecipitated with indicated antibodies, and proteins 
were separated with $7.5 \%$ SDS-PAGE for immunoblotting and visualized by a chemiluminescence reagent (Pierce).

\section{SUMOylation assay}

RIG-I and the SUMOylation enzymes were expressed in E.coli BL21 (DE3) individually and purified to homogeneity as previously described (Boggio et al., 2004). For the conjugation assay, $1 \mu \mathrm{g}$ SAE1/SAE2, $2 \mu \mathrm{g}$ Ubc9, $2 \mu \mathrm{g}$ SUMO1-GG and $0.5 \mu \mathrm{g}$ RIG-I in $15 \mu \mathrm{L}$ reaction buffer ( $20 \mathrm{mM}$ HEPES, $\mathrm{pH} 7.5,5 \mathrm{mM} \mathrm{MgCl}_{2}$ ) in the presence of $2 \mathrm{mM}$ ATP, $1 \mathrm{mM}$ DTT and $2 \mathrm{mM}$ NEM were incubated at $30^{\circ} \mathrm{C}$ for $3 \mathrm{~h}$. The reaction was terminated by adding $15 \mu \mathrm{L} 2 \times \mathrm{SDS}-\mathrm{PAGE}$ loading buffer and boiled at $95^{\circ} \mathrm{C}$ for $5 \mathrm{~min}$.

\section{RNAi assay}

The target sequence of Ubc9 (5'-GGGAAGGAGGCTTGTTTAAAC$3^{\prime}$ ) or its scrambled control sequence in a lentiviral vector LTV1 (Sui and Shi, 2005) was kindly provided by Prof. G. Sui (Wake Forest University School of Medicine, USA). Packaging lentiviruses were prepared as previously described (Rubinson et al., 2003). The lentiviral infection of HeLa cells was performed in the presence of $8 \mu \mathrm{g} / \mathrm{mL}$ polybrene (Sigma) for $4 \mathrm{~h}$ and the knockdown efficiency was measured $48 \mathrm{~h}$ post infection.

\section{RT-PCR}

Total RNA was isolated according to manufacturer's instruction with Trizol reagent (Invitrogen) and RT-PCR was performed using RTPCR kit (Promega) according to manufacturer's manual with the following primers: $h U b c 9$ forward, 5'-CGGAATTCTATGTCGGGGATСTCССTC-3'; hUbc9 reverse, 5'-CGGGGTACCTTATGAGGGCGCAAACTTC-3'; $h$ GAPDH forward, 5'-AAGCGCACGGGCATGGCCTT-3', hGAPDH reverse, 5'-AGGAGACCACCTGGTGCTCAG-3'. Quantitative real-time PCR reactions in a MyiQ cycler (Bio-Rad, USA) using SYBR Green I (Molecular Probes, USA) were performed exactly as described previously (Doyle et al. , 2002) with the following primers: human $\beta$-actin forward, 5'-GCGGGAAATCGTGCGTGACATT-3'; human $\beta$-actin reverse, 5 'GATGGAGTTGAAGGTAGTTTCGTG-3' (Lenz et al., 1994); SeV NP forward, 5'-TGCTGCCAAAGTTCACGAT-3'; SeV NP reverse, 5'ATAACTT GTCTGCATCATCA-3'.

\section{ACKNOWLEDGMENTS}

We thank Drs. S. Akira (Osaka University, Japan), G. Sui (Wake Forest University School of Medicine, USA), M. Gack (FriedrichAlexander University, Germany), G. Cheng (UCLA, USA) and X. Peng (Chinese Academy of Medical Sciences, China) for kindly providing us with various plasmid constructs, and our colleagues (G. Chen, D. Zheng, J. Jiang and X. Yang) for their critical reading and stimulating suggestions. This research was in part supported by grants from Chinese Academy of Sciences (Grant No. KSCX1-YW10), and the Ministry of Science and Technology (Grant Nos. 2006CB910901, 2007DFC30190, 2008ZX10001-002 and 2009CB522506) to H.T. The authors have no conflicting financial interests.

\section{ABBREVIATIONS}

CARD, caspase recruitment domain; Cardif, CARD adaptor inducing IFN- $\beta$; IFN, interferon; PRR, pattern recognition receptor; RIG-I, retinoic acid-inducible gene I; MDA5, melanoma-differentiationassociated gene 5; MEM, minimal essential medium; PAA, fetal bovine serum; RLH, RIG-I-like helicase; SeV, Sendai virus; SUMO, small ubiquitin-like modifier; TLR, Toll-like receptor; Ubi, ubiquitin; VSV, vesicular Stomatitis virus.

\section{REFERENCES}

Anckar, J., and Sistonen, L. (2007). SUMO: getting it on. Biochem Soc Trans 35, 1409-1413.

Arimoto, K., Konishi, H., and Shimotohno, K. (2008). UbcH8 regulates ubiquitin and ISG15 conjugation to RIG-I. Mol Immunol 45, 1078-1084.

Arimoto, K., Takahashi, H., Hishiki, T., Konishi, H., Fujita, T., and Shimotohno, K. (2007). Negative regulation of the RIG-I signaling by the ubiquitin ligase RNF125. Proc Natl Acad Sci U S A 104, 7500-7505.

Boggio, R., Colombo, R., Hay, R.T., Draetta, G.F., and Chiocca, S. (2004). A mechanism for inhibiting the SUMO pathway. Mol Cell 16, 549-561.

Comerford, K.M., Leonard, M.O., Karhausen, J., Carey, R., Colgan, S. P., and Taylor, C.T. (2003). Small ubiquitin-related modifier-1 modification mediates resolution of CREB-dependent responses to hypoxia. Proc Natl Acad Sci U S A 100, 986-991.

Cui, X.F., Imaizumi, T., Yoshida, H., Borden, E.C., and Satoh, K. (2004). Retinoic acid-inducible gene-I is induced by interferongamma and regulates the expression of interferon-gamma stimulated gene 15 in MCF-7 cells. Biochem Cell Biol 82, 401-405.

Desterro, J.M., Rodriguez, M.S., and Hay, R.T. (1998). SUMO-1 modification of IkappaBalpha inhibits NF-kappaB activation. Mol Cell 2, 233-239.

Desterro, J.M., Thomson, J., and Hay, R.T. (1997). Ubch9 conjugates SUMO but not ubiquitin. FEBS Lett 417, 297-300.

Doyle, S., Vaidya, S., O'Connell, R., Dadgostar, H., Dempsey, P., Wu, T., Rao, G., Sun, R., Haberland, M., Modlin, R., et al. (2002). IRF3 mediates a TLR3/TLR4-specific antiviral gene program. Immunity 17, 251-263.

Gack, M.U., Shin, Y.C., Joo, C.H., Urano, T., Liang, C., Sun, L., Takeuchi, O., Akira, S., Chen, Z., Inoue, S., et al. (2007). TRIM25 RING-finger E3 ubiquitin ligase is essential for RIG-I-mediated antiviral activity. Nature 446, 916-920.

Geiss-Friedlander, R., and Melchior, F. (2007). Concepts in sumoylation: a decade on. Nat Rev Mol Cell Biol 8, 947-956.

Gitlin, L., Barchet, W., Gilfillan, S., Cella, M., Beutler, B., Flavell, R.A., Diamond, M.S., and Colonna, M. (2006). Essential role of mda-5 in type I IFN responses to polyriboinosinic:polyribocytidylic acid and encephalomyocarditis picornavirus. Proc Natl Acad Sci U S A 103, 8459-8464.

Guo, B., and Cheng, G. (2007). Modulation of the interferon antiviral response by the TBK1/IKKi adaptor protein TANK. J Biol Chem 282, 11817-11826.

Guo, D., Li, M., Zhang, Y., Yang, P., Eckenrode, S., Hopkins, D., Zheng, W., Purohit, S., Podolsky, R.H., Muir, A., et al. (2004). A functional variant of SUMO4, a new I kappa B alpha modifier, is 
associated with type 1 diabetes. Nat Genet 36, 837-841.

Han, K.J., Jiang, L., and Shu, H.B. (2008). Regulation of IRF2 transcriptional activity by its sumoylation. Biochem Biophys Res Commun 372, 772-778.

Hay, R.T. (2005). SUMO: a history of modification. Mol Cell 18, 1-12. Hershko, A., and Ciechanover, A. (1998). The ubiquitin system. Annu Rev Biochem 67, 425-479.

Hornung, V., Ellegast, J., Kim, S., Brzozka, K., Jung, A., Kato, H., Poeck, H., Akira, S., Conzelmann, K.K., Schlee, M., et al. (2006). 5'-Triphosphate RNA is the ligand for RIG-I. Science 314, 994-997.

Huang, T.T., Wuerzberger-Davis, S.M., Wu, Z.H., and Miyamoto, S. (2003). Sequential modification of NEMO/IKKgamma by SUMO-1 and ubiquitin mediates NF-kappaB activation by genotoxic stress. Cell 115, 565-576.

Imaizumi, T., Hatakeyama, M., Yamashita, K., Yoshida, H., Ishikawa, A., Taima, K., Satoh, K., Mori, F., and Wakabayashi, K. (2004). Interferon-gamma induces retinoic acid-inducible gene-I in endothelial cells. Endothelium 11, 169-173.

Jounai, N., Takeshita, F., Kobiyama, K., Sawano, A., Miyawaki, A., Xin, K.Q., Ishii, K.J., Kawai, T., Akira, S., Suzuki, K., et al. (2007). The Atg5 Atg12 conjugate associates with innate antiviral immune responses. Proc Natl Acad Sci U S A 104, 14050-14055.

Kang, D.C., Gopalkrishnan, R.V., Lin, L., Randolph, A., Valerie, K., Pestka, S., and Fisher, P.B. (2004). Expression analysis and genomic characterization of human melanoma differentiation associated gene-5, mda-5: a novel type I interferon-responsive apoptosis-inducing gene. Oncogene 23, 1789-1800.

Kang, D.C., Gopalkrishnan, R.V., Wu, Q., Jankowsky, E., Pyle, A.M., and Fisher, P.B. (2002). mda-5: An interferon-inducible putative RNA helicase with double-stranded RNA-dependent ATPase activity and melanoma growth-suppressive properties. Proc Natl Acad Sci U S A 99, 637-642.

Kato, H., Sato, S., Yoneyama, M., Yamamoto, M., Uematsu, S., Matsui, K., Tsujimura, T., Takeda, K., Fujita, T., Takeuchi, O., et al. (2005). Cell type-specific involvement of RIG-I in antiviral response. Immunity 23, 19-28.

Kato, H., Takeuchi, O., Sato, S., Yoneyama, M., Yamamoto, M., Matsui, K., Uematsu, S., Jung, A., Kawai, T., Ishii, K.J., et al. (2006). Differential roles of MDA5 and RIG-I helicases in the recognition of RNA viruses. Nature 441, 101-105.

Kawai, T., Takahashi, K., Sato, S., Coban, C., Kumar, H., Kato, H., Ishii, K.J., Takeuchi, O., and Akira, S. (2005). IPS-1, an adaptor triggering RIG-I- and Mda5-mediated type I interferon induction. Nat Immunol 6, 981-988.

Kim, M.J., Hwang, S.Y., Imaizumi, T., and Yoo, J.Y. (2008). Negative feedback regulation of RIG-I-mediated antiviral signaling by interferon-induced ISG15 conjugation. J Virol 82, 1474-1483.

Kubota, T., Matsuoka, M., Chang, T.H., Tailor, P., Sasaki, T., Tashiro, M., Kato, A., and Ozato, K. (2008). Virus infection triggers SUMOylation of IRF3 and IRF7, leading to the negative regulation of type I interferon gene expression. J Biol Chem 283, 25660-25670.

Lenz, H.J., Danenberg, K., Schnieders, B., Goeker, E., Peters, G.J., Garrow, T., Shane, B., Bertino, J.R., and Danenberg, P.V. (1994). Quantitative analysis of folylpolyglutamate synthetase gene expression in tumor tissues by the polymerase chain reaction: marked variation of expression among leukemia patients. Oncol Res 6, 329-335.
Lim, K.L., Chew, K.C., Tan, J.M., Wang, C., Chung, K.K., Zhang, Y., Tanaka, Y., Smith, W., Engelender, S., Ross, C.A., et al. (2005). Parkin mediates nonclassical, proteasomal-independent ubiquitination of synphilin-1: implications for Lewy body formation. J Neurosci 25, 2002-2009.

Lin, R., Yang, L., Nakhaei, P., Sun, Q., Sharif-Askari, E., Julkunen, I., and Hiscott, J. (2006). Negative regulation of the retinoic acidinducible gene l-induced antiviral state by the ubiquitin-editing protein A20. J Biol Chem 281, 2095-2103.

Lin, X., Liang, M., Liang, Y.Y., Brunicardi, F.C., and Feng, X.H. (2003). SUMO-1/Ubc9 promotes nuclear accumulation and metabolic stability of tumor suppressor Smad4. J Biol Chem 278, 31043-31048.

Mattana, P., and Viscomi, G.C. (1998). Variations in the interferoninducing capacity of Sendai virus subpopulations. J Interferon Cytokine Res 18, 399405.

Melchior, F., Schergaut, M., and Pichler, A. (2003). SUMO: ligases, isopeptidases and nuclear pores. Trends Biochem Sci 28, 612-618.

Meylan, E., Curran, J., Hofmann, K., Moradpour, D., Binder, M., Bartenschlager, R., and Tschopp, J. (2005). Cardif is an adaptor protein in the RIG-I antiviral pathway and is targeted by hepatitis $C$ virus. Nature 437, 1167-1172.

Meylan, E., Tschopp, J., and Karin, M. (2006). Intracellular pattern recognition receptors in the host response. Nature 442, 39-44.

Rubinson, D.A., Dillon, C.P., Kwiatkowski, A.V., Sievers, C., Yang, L., Kopinja, J., Rooney, D.L., Zhang, M., Ihrig, M.M., McManus, M.T., et al. (2003). A lentivirus-based system to functionally silence genes in primary mammalian cells, stem cells and transgenic mice by RNA interference. Nat Genet 33, 401-406.

Saito, T., Hirai, R., Loo, Y.M., Owen, D., Johnson, C.L., Sinha, S.C., Akira, S., Fujita, T., and Gale, M., Jr.(2007). Regulation of innate antiviral defenses through a shared repressor domain in RIG-I and LGP2. Proc Natl Acad Sci U S A 104, 582-587.

Schwamborn, K., Knipscheer, P., van Dijk, E., van Dijk, W.J., Sixma, T.K., Meloen, R.H., and Langedijk, J.P. (2008). SUMO assay with peptide arrays on solid support: insights into SUMO target sites. J Biochem 144, 39-49.

Seth, R.B., Sun, L., Ea, C.K., and Chen, Z.J. (2005). Identification and characterization of MAVS, a mitochondrial antiviral signaling protein that activates NF-kappaB and IRF 3. Cell 122, 669-682.

Song, J., Durrin, L.K., Wilkinson, T.A., Krontiris, T.G., and Chen, Y. (2004). Identification of a SUMO-binding motif that recognizes SUMO-modified proteins. Proc Natl Acad Sci U S A 101, 14373-14378.

Steffan, J.S., Agrawal, N., Pallos, J., Rockabrand, E., Trotman, L.C., Slepko, N., Illes, K., Lukacsovich, T., Zhu, Y.Z., Cattaneo, E., et al. (2004). SUMO modification of Huntingtin and Huntington's disease pathology. Science 304, 100-104.

Sui, G., and Shi, Y. (2005). Gene silencing by a DNA vector-based RNAi technology. Methods Mol Biol 309, 205-218.

Taniguchi, T., and Takaoka, A. (2001). A weak signal for strong responses: interferon-alpha/beta revisited. Nat Rev Mol Cell Biol 2, 378-386.

Taniguchi, T., and Takaoka, A. (2002). The interferon-alpha/beta system in antiviral responses: a multimodal machinery of gene regulation by the IRF family of transcription factors. Curr Opin Immunol 14, 111-116. 
Xu, J., He, Y., Qiang, B., Yuan, J., Peng, X., and Pan, X.M. (2008). A novel method for high accuracy sumoylation site prediction from protein sequences. BMC Bioinformatics 9, 8.

Xu, L.G., Wang, Y.Y., Han, K.J., Li, L.Y., Zhai, Z., and Shu, H.B. (2005). VISA is an adapter protein required for virus-triggered IFNbeta signaling. Mol Cell 19, 727-740.

Yoneyama, M., and Fujita, T. (2008). Structural mechanism of RNA recognition by the RIG-I-like receptors. Immunity 29, 178-181.

Yoneyama, M., Kikuchi, M., Matsumoto, K., Imaizumi, T., Miyagishi, M., Taira, K., Foy, E., Loo, Y.M., Gale, M., Jr., Akira, S., et al. (2005). Shared and unique functions of the DExD/H-box helicases RIG-I, MDA5, and LGP2 in antiviral innate immunity. J Immunol 175,
2851-2858.

Yoneyama, M., Kikuchi, M., Natsukawa, T., Shinobu, N., Imaizumi, T., Miyagishi, M., Taira, K., Akira, S., and Fujita, T. (2004). The RNA helicase RIG-I has an essential function in double-stranded RNAinduced innate antiviral responses. Nat Immunol 5, 730-737.

Zhao, C., Denison, C., Huibregtse, J.M., Gygi, S., and Krug, R.M. (2005). Human ISG15 conjugation targets both IFN-induced and constitutively expressed proteins functioning in diverse cellular pathways. Proc Natl Acad Sci U S A 102, 10200-10205.

Zheng, L., Baumann, U., and Reymond, J.L. (2004). An efficient onestep site-directed and site-saturation mutagenesis protocol. Nucleic Acids Res 32, e115. 